\title{
ON UNIONS AND INTERSECTIONS OF SETS OF SYNTHESIS
}

\author{
T. K. MURALEEDHARAN AND K. PARTHASARATHY
}

(Communicated by J. Marshall Ash)

\begin{abstract}
Local techniques introduced by Saeki and Stegeman are employed to give conditions for unions and finite intersections of $S$-sets to be $S$-sets.
\end{abstract}

Using local techniques we study unions and intersections of sets of spectral synthesis ( $S$-sets). Beyond the well-known fact that the intersection of two $S$-sets need not be an $S$-set, there appears to be no known result about intersections. It is not known whether the union of two $S$-sets is an $S$-set, but there are some partial results (e.g., Saeki [2] and Warner [6]). We give simple sufficient conditions for finite intersections and (possibly infinite) unions of $S$-sets to be $S$-sets.

We generally follow the notation and terminology of Stegeman [4]. $A(G)$ denotes the Fourier algebra of a locally compact abelian group $G$. Suppose $x \in G, f, g \in A(G)$, and $I, J$ are ideals in $A(G)$. We write

(i) $f={ }_{x} g$ if $f$ and $g$ agree in some neighbourhood of $x$;

(ii) $f \in_{x} I$ if $f={ }_{x} g$ for some $g \in I$;

(iii) $I \subset_{x} J$ if $f \in_{x} J$ for every $f \in I$;

(iv) $I=_{x} J$ if $I \subset_{x} J$ and $J \subset_{x} I$.

For a closed subset $E$ of $G, I(E)$ and $J(E)$ denote the largest and smallest closed ideals, respectively, with hull E. $\Delta(E)$ ("the difference spectrum of $E$ " [3]) is the set of points of nonsynthesis of $E$,

$$
\Delta(E)=\left\{x \in G: I(E) \neq \neq_{x} J(E)\right\},
$$

so that $E$ is an $S$-set if and only if $\Delta(E)$ is empty. $\Delta(E)$ is a subset of $\partial E$, the boundary of $E$. It was used by Saeki [2] and was later systematically exploited in [4], with different notations. We also make frequent use of the following result proved by Stegeman [4].

Theorem A (Stegeman). If $E$ is a closed subset of $G$ such that $\Delta(E) \subset C \subset E$ for some $C$-set $C$, then $E$ is an $S$-set.

The basic idea of our approach is to get suitable relations between the difference spectra of the sets involved and their unions and intersections.

Received by the editors October 30, 1992 and, in revised form, July 27, 1993.

1991 Mathematics Subject Classification. Primary 43A45, 43A46.

Key words and phrases. $S$-set, $C$-set, difference spectrum.

The first author is a CSIR Research Fellow. 
1. Lemma. Let $E_{1}, E_{2}$ be closed subsets of $G$. Then

(i) $\Delta\left(E_{1} \cap E_{2}\right) \subset \Delta\left(E_{1}\right) \cup \Delta\left(E_{2}\right) \cup\left(\partial E_{1} \cap \partial E_{2}\right)$,

(ii) $\Delta\left(E_{1}\right) \cup \Delta\left(E_{2}\right) \subset \Delta\left(E_{1} \cup E_{2}\right) \cup\left(E_{1} \cap E_{2}\right)$.

Proof. Let $x \in \Delta\left(E_{1} \cap E_{2}\right) \subset \partial\left(E_{1} \cap E_{2}\right)$. Suppose $x \notin \partial E_{1} \cap \partial E_{2}$. Since $\partial\left(E_{1} \cap E_{2}\right) \subset \partial E_{1} \cup \partial E_{2}$, as is easily checked, we have $x \in \partial E_{1}$ or $x \in \partial E_{2}$, but not both. We prove that $x \in \Delta\left(E_{i}\right)$ if $x \in \partial E_{i}, i=1,2$.

Suppose $x \in \partial E_{1}$. Now $x \notin \partial E_{2}$ and $x \in E_{2}$ imply that there is a neighbourhood $V$ of $x$ with $V \subset E_{2}$. Choose $k \in A(G)$ with $k=1$ in a neighbourhood of $x$ and supp $k \subset V$. Since $x \in \Delta\left(E_{1} \cap E_{2}\right)$, there is an $f \in I\left(E_{1} \cap E_{2}\right)$ such that $f \notin_{x} J\left(E_{1} \cap E_{2}\right)$. Then $f k \in I\left(E_{1}\right)$. If $x \notin \Delta\left(E_{1}\right)$, then $f k \in_{x} J\left(E_{1}\right)$, so $f==_{x} f k \in J\left(E_{1}\right) \subset J\left(E_{1} \cap E_{2}\right)$, a contradiction.

Thus $x \in \partial E_{1}$ implies $x \in \Delta\left(E_{1}\right)$. Similarly, if $x \in \partial E_{2}$, then $x \in \Delta\left(E_{2}\right)$ and (i) is proved.

The proof of (ii) is similar but simpler: one proves that if $x \in \Delta\left(E_{1}\right)$ and $x \notin E_{2}$, then $x \in \Delta\left(E_{1} \cup E_{2}\right)$.

Our first application of Lemma 1 is the following result on intersections. It shows, for instance, that an annulus is an $S$-set.

2. Theorem. Let $E_{1}, E_{2}$ be $S$-sets. If there is a $C$-set $C$ such that $\partial E_{1} \cap \partial E_{2} \subset$ $C \subset E_{1} \cap E_{2}$, then $E_{1} \cap E_{2}$ is an $S$-set. In particular, the conclusion holds if $\partial E_{1} \cap \partial E_{2}=\varnothing$.

Proof. By (i) of Lemma 1

$$
\Delta\left(E_{1} \cap E_{2}\right) \subset \partial E_{1} \cap \partial E_{2} \subset C \subset E_{1} \cap E_{2},
$$

so the assertion is a consequence of Theorem $\mathrm{A}$.

3. Remark. If $E_{1}, E_{2}, E_{3}$ are $S$-sets with $\partial E_{1} \cap \partial E_{2} \cap \partial E_{3}=\varnothing$, it is not necessary that $E_{1} \cap E_{2} \cap E_{3}$ is an $S$-set. Take, for example, $E_{1}=G=\mathbb{R}^{3}$, $E_{2}=$ the closed unit ball, and $E_{3}=$ the complement of the open unit ball. For a finite collection $\left\{E_{i}\right\}$ of $S$-sets, the correct version is that if $\partial E_{i} \cap \partial E_{j}=\varnothing$ for $i \neq j$, then $\cap E_{i}$ is an $S$-set. This again is no longer true for infinite collections: take $E_{1}=$ the closed unit ball in $\mathbb{R}^{3}$ and $E_{n}=\left\{x \in \mathbb{R}^{3}:\|x\| \geq 1-\frac{1}{n}\right\}, n>1$.

We now come to results on unions. We first mention the following result of Saeki [2].

4. Theorem (Saeki). If $E_{1}, E_{2}$ are $S$-sets and if there is a $C$-set $C$ such that

$$
\partial\left(E_{1}\right) \cap \partial\left(E_{2}\right) \cap \partial\left(E_{1} \cup E_{2}\right) \subset C \subset E_{1} \cup E_{2},
$$

then $E_{1} \cup E_{2}$ is an $S$-set.

5. Remark. Theorem 4 is also stated as one part of Theorem $4^{\prime}$ in Warner [6], the other part being a converse, namely, if $E_{1} \cup E_{2}$ is an $S$-set and if there is a $C$-set $C$ satisfying the condition mentioned in Theorem 4, then $E_{1}$ and $E_{2}$ are $S$-sets. However, it is easy to see that this converse statement is false. Just take $E_{1}=S^{2}$ and $E_{2}=$ any closed convex set (e.g., a ball) in $\mathbb{R}^{3}$ containing $E_{1}$ in its interior; then $\partial E_{1} \cap \partial E_{2}=\varnothing, E_{1} \cup E_{2}=E_{2}$ is an $S$-set, but $E_{1}$ is not an $S$-set. The same example shows also that Warner's Lemma $3^{\prime}$ [6] is not true. We, however, have the following 'if and only if' result. 
6. Theorem. Let $E_{1}, E_{2}$ be closed subsets of $G$. Suppose there are $C$-sets $C_{1}, C_{2}$ such that

$$
\partial E_{1} \cap E_{2} \subset C_{1} \subset E_{1}, \quad E_{1} \cap \partial E_{2} \subset C_{2} \subset E_{2} .
$$

Then $E_{1} \cup E_{2}$ is an $S$-set if and only if $E_{1}$ and $E_{2}$ are $S$-sets.

Proof. Since $\Delta\left(E_{i}\right) \subset \partial E_{i}$, it follows from Lemma 1(ii) that $\Delta\left(E_{1}\right) \subset$ $\Delta\left(E_{1} \cup E_{2}\right) \cup\left(\partial E_{1} \cap E_{2}\right)$ and similarly for $E_{2}$. Theorem $\mathrm{A}$ now gives one half of the result, while the other half is a consequence of Theorem 4 (recalling that the union of two $C$-sets is a $C$-set).

It is well known that a closed countable union of $C$-sets is a $C$-set. Here is a result for infinite unions of $S$-sets.

7. Lemma. Let $\left\{E_{i}\right\}$ be a collection of mutually disjoint closed sets in G. Suppose that, for each $j, \bigcup_{i \neq j} E_{i}$ is closed. Then

(i) $\Delta\left(\bigcup E_{i}\right) \subset \bigcup \Delta\left(E_{i}\right)$,

(ii) $\Delta\left(E_{j}\right) \subset \Delta\left(\bigcup E_{i}\right)$ for each $j$.

Proof. Let $x \in \Delta\left(\bigcup E_{i}\right)$. Then $x \in E_{j}$ for a unique $j$. We prove $x \in \Delta\left(E_{j}\right)$. Choose a neighbourhood $V$ of $x$ such that $\bar{V} \cap\left(\bigcup_{i \neq j} E_{i}\right)=\varnothing$, and then choose a $k \in A(G)$ such that $k=1$ near $x$ and $k=0$ off $V$. Since $x \in \Delta\left(\bigcup E_{i}\right)$, there is an $f \in I\left(\bigcup E_{i}\right)$ with $f \notin_{x} J\left(\bigcup E_{i}\right)$.

Assume $x \notin \Delta\left(E_{j}\right)$. Then there is a $g \in J\left(E_{j}\right)$ with $f==_{x} g$. But $g k \in$ $J\left(\bigcup E_{i}\right)$, so that $f={ }_{x} f k==_{x} g k \in J\left(\bigcup E_{i}\right)$, a contradiction. Hence (i) is proved. The proof of (ii) does not need any new ideas, and we omit it.

8. Theorem. Let $\left\{E_{i}\right\}$ be a collection of mutually disjoint closed sets in $G$ satisfying the condition that $\bigcup_{i \neq j} E_{i}$ is closed for each $j$. Then $\bigcup E_{i}$ is an $S$-set if and only if each $E_{i}$ is an $S$-set.

Proof. Immediate from Lemma 7.

Using the fact that a closed set which is a countable union of $C$-sets is a $C$-set, arguments similar to the above give the following result.

9. Theorem. Let $\left\{E_{i}\right\}$ be a collection of closed subsets of $G$ satisfying the following conditions:

(a) $E_{i} \cap E_{j}=\varnothing, i \neq j$, except for countably many $i, j$.

(b) $E_{i} \cap E_{j}$ is a $C$-set for all $i, j, i \neq j$.

(c) $\bigcup_{i \neq j} E_{i}$ is closed for each $j$.

(d) $\bigcup_{i, j, i \neq j}\left(E_{i} \cap E_{j}\right)$ is closed.

Then $U E_{i}$ is an $S$-set if and only if each $E_{i}$ is an $S$-set.

10. Corollary. Let $\left\{E_{i}\right\}$ be a collection of $S$-sets in $G$ satisfying (a), (b) and (d) of Theorem 9. In place of (c), suppose that $C=\bigcup_{j}\left\{\overline{\bigcup_{i \neq j} E_{i}} \backslash \bigcup_{i \neq j} E_{i}\right\}$ is a $C$-set. Then $\overline{\cup E_{i}}$ is an S-set.

Proof. $F_{i}=E_{i} \cup C$ are $S$-sets satisfying the conditions of Theorem 9 and $\overline{\cup E_{i}}=\bigcup F_{i}$.

11. Remark. It is easy to construct examples of $S$-sets using our results, e.g., the Hawaiian ear ring in $\mathbb{R}^{2}$ [5, pp. 111-112]. Lemma 4.3.7 of [1], which says 
that $\left(\bigcup E_{n}\right) \cup\{0\}$ is an $S$-set in $\mathbb{R}$, where each $E_{n} \subset\left(\frac{1}{n+1}, \frac{1}{n}\right)$ is an $S$-set, can also be deduced from the results given above.

\section{ACKNOWLEDGMENT}

The authors thank the referee for useful remarks.

\section{REFERENCES}

1. C. C. Graham and O. C. McGehee, Essays in commutative harmonic analysis, SpringerVerlag, Berlin, 1979.

2. S. Saeki, Spectral synthesis for Kronecker sets, J. Math. Soc. Japan 21 (1969), 549-563.

3. D. L. Salinger and J. D. Stegeman, Difference spectra of ideals for non-metrizable groups, J. London Math. Soc. (2) 26 (1982), 531-540.

4. J. D. Stegeman, Some problems on spectral synthesis, Proceedings, Harmonic Analysis (Iraklion, 1978), Lecture Notes in Math., vol. 781, Springer-Verlag, Berlin, 1980, pp. 194-203.

5. C. T. C. Wall, A geometric introduction to topology, Addison-Wesley, Reading, MA, 1972.

6. C. R. Warner, A class of spectral sets, Proc. Amer. Math. Soc. 57 (1976), 99-102.

Ramanujan Institute, University of Madras, Madras 600 005, India 